\title{
Selective Partitioning of (Biomacro)molecules in the Crowded Environment of Double-Hydrophilic Block Copolymers
}

\author{
Rafael B. Lira, Jochen Willersinn, Bernhard V. K. J. Schmidt,* and Rumiana Dimova*
}

Cite This: Macromolecules 2020, 53, 10179-10188

Read Online

ABSTRACT: The phase separation in aqueous solutions of a double-hydrophilic block copolymer (DHBC) at concentrations mimicking the crowded environment in cells and its performance as a potential protein purification system are investigated. Similar to processes of self-coacervation, the coexistence of aqueous polymer-rich and polymer-depleted microscopic phases is observed and the partitioning of various species in them is investigated. Whereas small molecules, poly(ethylene glycol), and dextran can moderately partition in both phases,

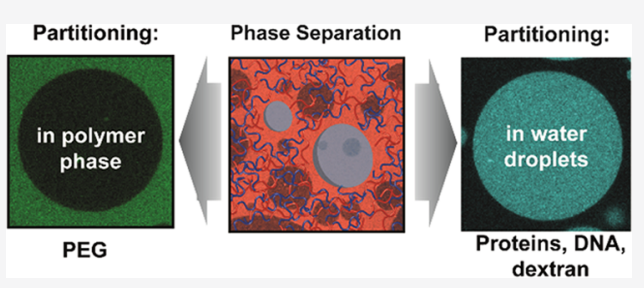
proteins and DNA are strongly enriched in the polymer-depleted phase, offering a pathway for biomacromolecule purification. While aqueous two-phase systems based on a pair of polymers are also used for purification of biological material, DHBC has the remarkable advantage that the proteins and DNA segregate in the polymerdepleted phase, enhancing and facilitating purification. Future optimization of DHBC chemistry may offer higher partition efficiency and a way for large-scale applications.

\section{INTRODUCTION}

Block copolymers and their self-assembly have been explored in various realms of science. Amphiphilic block copolymers can self-assemble, for example, into so-called polymersomes ${ }^{1,2}$ nowadays extensively utilized in biomedical applications and drug delivery. ${ }^{3,4}$ Their assembly is driven by the hydrophobic effect. On the contrary, double-hydrophilic block copolymers (DHBCs; i.e., polymers with only hydrophilic blocks but no hydrophobic blocks) and their organization and phase behavior are dominated rather by the hydrophilic effect based on the difference in affinity or solubility of the different blocks in water, leading to phase separation in a considerable number of cases. 5 Contrary to traditional amphiphilic polymers, DHBCs with fully water-soluble blocks allow potentially higher biocompatibility and degradability. They have been investigated frequently as stimuli-responsive materials ${ }^{7-9}$ and as platforms for mineralization of inorganic compounds. ${ }^{10,11}$ However, the majority of studies have focused on systems of low polymer concentration (from a few ppm to approximately $1 \mathrm{mg} / \mathrm{mL}$ ) (see, e.g., refs 5 and 12-14). Their behavior in the intermediate concentration regime remains rather unexplored. A report by Taubert et al. demonstrated the presence of mesoscale water-in-water phase separation ${ }^{15}$ in poly(ethylene glycol)- $b$-poly(2-methyl-2-oxazoline) (PEG- $b$ PMOXA) solutions. This was evidenced by the appearance of optically detectable droplets with birefringence characteristics (demonstrating long-scale order). Indeed, such phase separation was observed also in solutions of poly(ethylene glycol)- $b$-dextran (PEG- $b$-dextran), PEG- $b$-pullulan (PEG- $b$ Pull), and dextran- $b$-poly(sarcosine) where the aggregates were referred to as giant vesicles, ${ }^{16}$ which might need reconsideration as these structures could actually represent droplets as those reported by Taubert et al. ${ }^{15}$ and our findings described below.

The phase separation behavior in DHBC solutions at high polymer concentrations is similar to that in aqueous two-phase systems (ATPSs) observed for solutions of hydrophilic homopolymer couples (representing the building blocks of DHBCs), such as PEG and dextran. ${ }^{17-20}$ ATPS systems have been extensively studied and found application, among others, in reversible microcompartmentalization ${ }^{21,22}$ of biomolecules such as proteins and nucleic acids, as well as for modulating compartmentalization of synthetic cells (vesicles or droplets) ${ }^{23-27}$ and shaping membranes. ${ }^{28-30}$ As we will demonstrate here for the first time, phase separation in DHBCs can also be used for protein and nucleic acid concentration with the advantage that these macromolecules can be concentrated in the polymer-poor phase, which significantly improves their purification as contamination with polymers is reduced. The system we explore may also offer a pathway of studying and understanding the acting mechanisms in more complex protein condensation and coacervation processes as nowadays extensively investigated in the field of cellular organization and stability of membrane-less organelles. ${ }^{31,32}$

Received: August 11, 2020

Revised: October 12, 2020

Published: November 15, 2020 


\section{EXPERIMENTAL SECTION}

2.1. Materials. Azobis(isobutyronitrile) (99\%, Sigma-Aldrich) was recrystallized twice from methanol. Dichloromethane (99.9\%, Acros Organics) and acetone (99\%, J.T. Baker) were stored over molecular sieves (3 $\AA$ ) prior to use. $N, N$-Dimethylacrylamide (DMA; 99\%, TCI) was passed over neutral aluminum oxide prior to use. Millipore water was obtained from an Integra UV plus pure water system by SG Water (Germany). Ammonium chloride (99\%, Roth KG), ascorbic acid (98\%, Alfa Aesar), bovine albumin-FITC (Sigma-Aldrich; M.W. $\sim 66.4 \mathrm{kDa}$ ), 2-bromo-2-methylpropanoic acid (98\%, Sigma-Aldrich), 3-bromo-1-propanol (97\%, Sigma-Aldrich), calcein (Sigma-Aldrich), carbon disulfide (anhydrous, 99\%, Sigma-Aldrich), chloromethyl polystyrene resin $(2.4 \mathrm{mmol} / \mathrm{g}$, TCI), copper sulfate $(99 \%$, Carl Roth), dimethylsulfoxide (DMSO; Acros, extra dry, 99\%), dextranFITC (10 and $20 \mathrm{kDa}$, Sigma-Aldrich), dextran from Leuconostoc mesenteroides (molar mass between 400 and $500 \mathrm{~kg} / \mathrm{mol}$, SigmaAldrich), $N, N^{\prime}$-dicyclohexylcarbodiimide (99\%, Sigma-Aldrich), diethylether (analytical grade, Sigma-Aldrich), $N, N$-dimethylaminopyridine (99\%, Sigma-Aldrich), dodecanethiol (98\%, Alfa Aesar), ethyl acetate (analytical grade, Chem Solute), ethylenediamine resin (polymer-bound, 4.0-5.7 mmol/g, Sigma-Aldrich), fluorescein isothiocyanate (FITC; 90\%, Sigma-Aldrich), hexane (analytical grade, Fluka), hexylamine ( $>99 \%$, Fluka), hydrochloric acid (fuming, Carl Roth), magnesium sulfate (dried, Fisher Scientific), methanol (analytical grade, Fisher Scientific), $N, N, N^{\prime}, N^{\prime \prime}, N^{\prime \prime}$-pentamethyldiethylenetriamine (98\%; Sigma-Aldrich), poly(ethylene glycol) diamine $\left(\mathrm{NH}_{2}\right.$-PEG-NH ${ }_{2} ; 2 \mathrm{k}, 10 \mathrm{k}$, and $20 \mathrm{k}$, analytical grade, Sigma-Aldrich), poly(ethylene glycol) (PEG; molar mass, $8 \mathrm{~kg} / \mathrm{mol}$; Sigma-Aldrich), potassium phosphate (Sigma-Aldrich), propargylamine (98\%, SigmaAldrich), pullulan (Pull; pure, TCI), rhodamine B isothiocyanate (99\%, Sigma-Aldrich), sodium azide (>99.5\%, Fluka), sodium bicarbonate ( $>99 \%$, Fluka), sodium cyanoborohydride (95\%, SigmaAldrich), and sulforhodamine B sodium salt (SRB; Sigma-Aldrich) were used as received. Lipids (POPC, POPG, DOTAP, and DPPENBD) were purchased from Avanti Polar Lipids (Alabaster, AL). The Vps24 protein (M.W. $\sim 31 \mathrm{kDa}$ ) was a gift from Y. Avalos (Institute for Bioengineering of Catalonia, Barcelona, Spain) and details of its purification are found elsewhere. ${ }^{33}$ GFP-histidine tag (GFP-his; M.W. $\sim 28.7 \mathrm{kDa}$ ) was a gift from S. Wegner (Max Planck Institute for Polymer Research, Mainz, Germany) and the Cy5-labeled DNA $(6.5-7 \mathrm{kDa})$ was a gift from R. Knorr (Max Planck Institute of Colloids and Interfaces, Potsdam, Germany). Polystyrene particles with various diameters as indicated in the main text were obtained from Polysciences Inc. (Warrington, PA) at a concentration of the stocks between 2.59 and $2.72 \%(\mathrm{w} / \mathrm{v})$. The 20 and $80 \mathrm{~nm}$ gold nanoparticles were obtained respectively from Sigma-Aldrich and BBI Solutions.

2.2. Polymer Synthesis, Labeling, Liposome Extrusion, and Sample Preparation. Pullulan- $b$-poly $(N, N$-dimethylacrylamide $)$ (Pull-b-PDMA) and rhodamine-labeled DHBC (Pull-b-PDMA-Rh) were synthesized according to the literature ${ }^{34}$ (for further details on synthesis and characterization, see the original reference $\left.{ }^{34}\right)$. A lyophilized polymer $\left(M_{\mathrm{n}}=21,500 \mathrm{~g} / \mathrm{mol}, \mathrm{D}=1.9\right.$, according to pullulan standards) was weighted in a glass vial and solubilized with an equivalent volume of Milli- $Q$ water to prepare a typical $20 \mathrm{wt} \%$ polymer concentration. To ensure complete solubilization, the polymer solution was left overnight under occasional manual agitation. Aliquots of this stock solution were sampled with an extra addition of water if a lower concentration of polymers was used. Samples were used within a week. When required, the polymer samples were supplemented with 0.02 wt \% labeled polymer final concentration $(0.1 \%$ of all the polymers were labeled) of Pull-bPDMA-Rh; the labeled polymers had one fluorescent group per polymer chain. Before pipetting a sample for observation, the stock vial was tapped to ensure mixing of the two phases and droplet formation. An aliquot of $10 \mu \mathrm{L}$ was placed between clean glass coverslips separated by a thin spacer $(0.5 \mathrm{~mm})$, forming a chamber, and brought for microscopy imaging. After pipetting the solution, depositing it on the coverslip, and waiting for $1 \mathrm{~h}$, the heavy polymer- rich phase settles to the bottom (with some droplets of the polymerpoor phase), representing the continuous phase in the images shown in the figures. All experiments were performed at room temperature $\left(23.9^{\circ} \mathrm{C}\right)$.

PEG-FITC was synthesized according to the literature. ${ }^{20}$ Aminefunctionalized resin was added to scavenge free unreacted FITC molecules. Then, purification was performed via dialysis to remove residual fluorescein.

Multilamellar liposomes (MLVs) were prepared by depositing a 3 $\mathrm{mM}$ lipid solution containing the respective lipid mixture in chloroform in a glass vial. ${ }^{35}$ The solutions additionally contained $0.5 \mathrm{~mol} \%$ of the fluorescent lipid DPPE-NBD. Chloroform was evaporated by a stream of $\mathrm{N}_{2}$ and any residue was further removed by placing the sample in vacuum for $2 \mathrm{~h}$ in the dark to form a lipid film. This film was hydrated with Milli-Q water and vigorously vortexed for $\sim 30$ s until MLs were formed.

For all nano- and microparticles, a $5 \mu \mathrm{L}$ aliquot of the particles stock was added to $10 \mu \mathrm{L}$ of Pull-b-PDMA solution supplemented with $0.02 \mathrm{wt} \%$ labeled polymer.

2.3. Image Analysis of Droplets. To observe droplet coalescence events, the solutions were observed at a full frame $(2048 \times 1952$ pixels $)$ using a high-speed Phantom camera (Ametek, Mahwah, NJ) at 100 frames per second with a pixel size of $0.71 \mu \mathrm{m}$. The shape relaxation after coalescence was measured using the inhouse built program previously described in Gracià et al. ${ }^{36}$ In short, the contour of the droplets was located with subpixel resolution, and the droplet dimensions were tracked over time. The temporal changes in the droplet aspect ratio upon coalescence were measured for many individual droplet-droplet coalescence events in the absence and presence of "guest" molecules.

2.4. Microscopy Imaging and Fluorescence Recovery after Photobleaching (FRAP). Phase contrast was used to watch droplet coalescence. It was performed on a Zeiss Axiovert 200 (Jena, Germany) phase contrast microscope equipped with a $40 \times$ objective (NA 0.6) and images were acquired at 100 frames per second with a v2512 Phantom camera. A Leica SP8 microscope was used for confocal microscopy. Images were acquired with a water-immersion $63 \times$ objective (NA 1.2) in the bidirectional mode with $512 \times 512$ pixel resolution and $400 \mathrm{~Hz}$ scanning speed and two line averages. The green probes calcein, GFP, and FITC-labeled polymers were excited with an argon 488 laser and emission was detected in the range of $490-545 \mathrm{~nm}$. The red dyes SRB and rhodamine-polymer (Pull- $b$-PDMA-Rh) were excited with a diode-pumped solid-state laser at $561 \mathrm{~nm}$ and emission was detected in the range of 565-620 $\mathrm{nm}$. The free or protein-labeled far-red Atto-647 dye was excited with a $\mathrm{HeNe} 633$ laser line and emission was detected in the $635-690 \mathrm{~nm}$ range. Image analysis was performed using ImageJ (NIH, USA) and Leica LAS X (Jena, Germany) software.

Fluorescence recovery after photobleaching (FRAP) was performed on the same setup with active FRAP booster tool to induce deeper photobleaching. For each of the respective dyes, the laser line was the same as that for imaging (see above). FRAP was performed with $128 \times 128$ pixel resolution, $1400 \mathrm{~Hz}$ scanning speed, and no line average. Ten prebleaching images with attenuated laser were acquired as a reference and the laser was switched to its maximum power (eight iterations) after which the laser was back to attenuated intensity until full recovery. The defined circular bleached region of interest was 8 $\mu \mathrm{m}$ in diameter. With these defined settings, images are taken every $55 \mathrm{~ms}$. FRAP recovery curves were analyzed using the simplified equations as introduced by Kang et al., ${ }^{37,38}$ where the diffusion coefficient $D$ is given by $D=\frac{r_{\mathrm{n}}^{2}+r_{\mathrm{e}}^{2}}{8 t_{1 / 2}}$. Here, $t_{1 / 2}$ is the time at which the intensity has recovered to half of its maximum, $r_{\mathrm{n}}$ is the userdefined nominal radius of photobleaching, and $r_{\mathrm{e}}$ is the calculated effective radius from the first image after photobleaching according to $f(x)=1-K \exp \left(-2 x^{2} / r_{\mathrm{e}}^{2}\right)$, where $K$ is the photobleaching depth (see Figure S4A for an example fit of a bleached area). Recovery curves were not corrected for photobleaching as it accounts for less than $1 \%$. 
2.5. Dynamic Light Scattering. The polymer hydrodynamic radius was determined using dynamic light scattering (DLS) (see Figure S6). DLS was performed using an ALV-7004 Multiple Tau Digital Correlator in combination with a CGS-3 Compact Goniometer and HeNe laser (Polytec, $34 \mathrm{~mW}, \lambda=633 \mathrm{~nm}$ at $\theta=$ $90^{\circ}$ setup for DLS). Toluene was used as an immersion liquid and sample temperatures were adjusted to $25{ }^{\circ} \mathrm{C}$. Hydrodynamic radii were determined from fitting autocorrelation functions using the CONTIN algorithm.

\section{RESULTS AND DISCUSSION}

3.1. Phase Separation in Aqueous Solutions of DHBC. Here, we investigate the DHBC Pull-b-poly $(N, N$-dimethylacrylamide) (Pull-b-PDMA), which is a combination of a bioderived polysaccharide block and reversible deactivation radical polymerization-derived block (see Figure 1A). Both

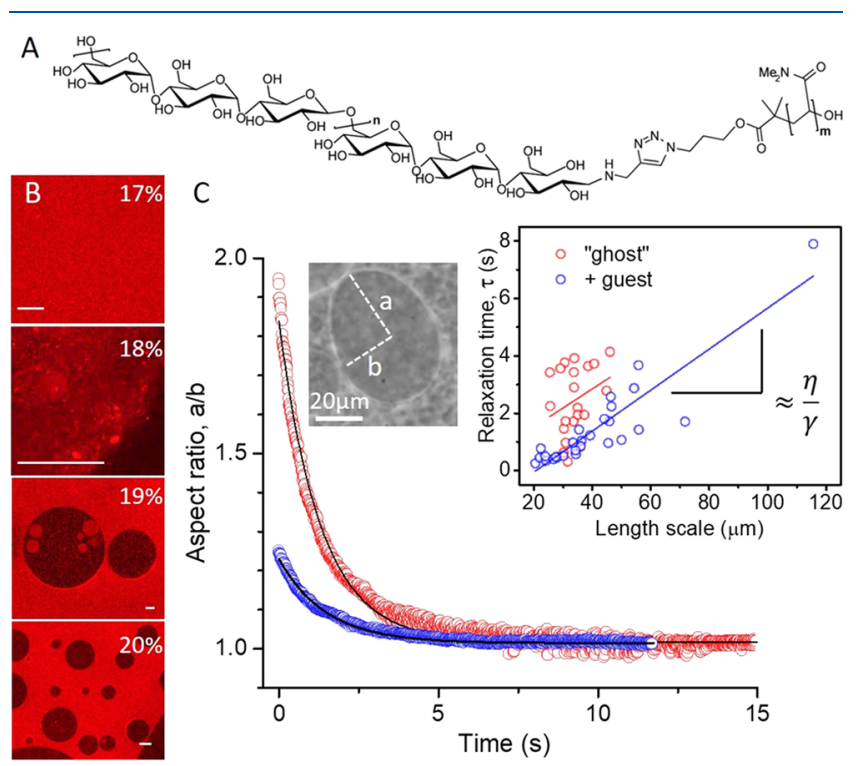

Figure 1. Solutions of Pull-b-PDMA double-hydrophilic block copolymer phase-separate into two fluid aqueous phases at high concentration. (A) Molecular structure of Pull-b-PDMA (average degree of polymerization: $n=29, m=169$ ). (B) Typical confocal images of polymer solutions at increasing polymer weight fractions. All conditions contain 0.02 wt \% Pull- $b$-PDMA-Rh. Scale bars correspond to $10 \mu \mathrm{m}$. (C) Typical relaxation curves in $20 \mathrm{wt} \%$ Pull-bPDMA solutions for droplet coalescence determined from the droplet aspect ratio $a / b$ (see image inset) for "ghost" droplets (polymerdepleted and protein-free, red) and droplets in a solution containing the guest protein Vps24 at a total concentration of $30 \mu \mathrm{M}$ (blue). Exponential fits (black curves) are used to determine the coalescence relaxation times $\tau$ (see text for details) shown in the inset graph as a function of the final droplet diameter. Lines are linear fits from which the inverse capillary velocity $(\eta / \gamma)$ is obtained.

blocks feature opposing hydrogen bonding characteristics, which enables concentration-dependent self-assembly in an aqueous environment. In the low-concentration range between 2 and $10 \mathrm{wt} \%$, Pull- $b$-PDMA forms shell-like structures of 100-200 nm as observed with cryo-scanning electron microscopy; ${ }^{34}$ these solutions appear to be optically clear. At higher concentrations, the polymer solution demixes into mesoscale structures directly observed as droplets under the microscope. Small fractions of Rhodamine end-labeled polymer (Pull-b-PDMA-Rh; see Section 2.1, Materials) added to the samples show that the polymer is enriched in the continuous phase (Figure 1B). The onset of phase separation is around 19 wt \%, below which only submicroscopic structures exist. The kinetics of phase separation is similar to that observed in conventional PEG-dextran systems (for example, at $20 \mathrm{wt} \%$ polymer concentration, phase separation is completed within a couple of hours and can be sped up by centrifugation). The phase behavior of DHBC is governed by the hydrophilic effect associated with the different solubilities of the blocks in water. ${ }^{5,6}$ The formation of a polymer-rich phase is most likely also driven by interactions of the different blocks in the DHBC. The PDMA block acts as a hydrogen bond acceptor, while the Pull block acts as a hydrogen bond donor, leading to the polymer phase separation similar to that in biomolecular condensates. ${ }^{31}$ Tests for bulk phase separation at higher Pull-b-PDMA concentrations show that the polymer-rich phase is denser, and high temperatures can suppress the phase separation (Figure S1), presumably shifting the binodal to higher polymer concentrations as entropy favors homogeneous mixing. ${ }^{39}$ If not mentioned otherwise, all following experiments were carried out at room temperature with $20 \mathrm{wt} \%$ polymer, of which $0.1 \%$ (i.e., $0.02 \mathrm{wt}$ $\%)$ was fluorescently labeled. Measurements of the polymer concentration in the two phases based on freeze-drying were avoided as they require large amounts of sample to achieve reasonable precision. We then attempted to assess the polymer concentration in the continuous and droplet phases from the absolute intensity of Pull- $b$-PDMA-Rh evaluated and an intensity calibration curve of the fluorescent polymer at preset concentrations. However, we observed differences in droplet intensity resulting from out-of-focus fluorescence and variation of size and shape due to partial wetting of the substrate by the droplets (see scatter in the data for the droplets in Figure S2). We thus evaluated the polymer partitioning from measurements of the volume fractions of the two phases and their relatively averaged fluorescence intensities (Figure S2). This yielded the polymer concentration in the dispersed phase (droplets) of approximately $15 \mathrm{wt} \%$, while in the continuous phase, it is enriched and is roughly 47 wt \%. The latter is close to typical concentrations in the crowded media of the cell cytosol where macromolecule concentrations can be as high as 30 wt \% and can increase under stress conditions such as hyperosmotic shifts. ${ }^{40}$ Similarly, we observe phase separation reminiscent of the formation of liquid condensates in the cytosol. ${ }^{41}$ The concentration-dependent condensation, which we observe, is reversible: an increase in temperature (Figure $\mathrm{S1}$ ) or polymer dilution results in homogeneous solutions. The phases are characterized by large permeability to dispersed molecules. Fluorescence recovery after photobleaching (FRAP) shows that small molecules freely penetrate and diffuse in and across the compartments with no significant barriers to diffusion (Figure S3A). In addition, upon contact, the droplets coalesce, leading to immediate mixing of their content (Figure 1C and Figure S3B), confirming their fluid nature. Taken together, droplet coalescence and free diffusion of molecules demonstrate liquid-liquid phase separation in this system rather than assembly into shells or vesicles.

3.2. Interfacial Tension and Viscosity of Phases. Upon coalescence, liquid droplets relax to a spherical shape to minimize the interfacial energy. During the relaxation process, we do not observe toroidal-like deformations in the neck region of the coalescing droplets, which suggests that the viscosities of the two phases do not differ much. ${ }^{42}$ The characteristic relaxation time $(\tau)$ depends linearly on the size of the coalescing droplets. The slope of this dependence yields 
the inverse capillary velocity $\eta / \gamma$, where $\eta$ is the viscosity of the droplet phase and $\gamma$ is the interfacial tension. To determine $\eta / \gamma$, the shape relaxation of droplet upon coalescence was measured, as previously done to characterize the physical properties of protein-rich droplets (also called condensates) in vivo and in vitro. ${ }^{32,43}$ The relaxation curve for two coalescing water-rich droplets in the Pull-b-PDMA-rich surrounding follows an exponential behavior, as expected (Figure 1C, red data). This coalescence dynamics of ghost droplets was compared to the coalescence of droplets containing a guest molecule. As a prototype guest, we used the protein Vps24 $(\sim 32 \mathrm{kDa})$, a member of the ESCRT-III machinery (see, e.g., ref 33). This protein exhibits very strong enrichment into the polymer-depleted phase, i.e., in the droplets (see below and Figure 3). The relaxation of Vps24-enriched droplets is also exponential and hence also characteristic of a fluid phase (Figure 1B, blue data). The obtained values for $\eta / \gamma$ for both systems were similar, with $0.066 \pm 0.005$ and $0.071 \pm 0.007 \mathrm{~s} /$ $\mu \mathrm{m}$ for ghost droplets and for droplets with the guest protein, respectively, where the errors were obtained from the fit. The $\eta / \gamma$ values are three orders of magnitude lower than those for RNA-protein droplets, ${ }^{32,43,44}$ which shows that Pull-b-PDMAdepleted droplets, with or without the guest protein, are significantly less viscous (and/or with higher interfacial tension) than typical droplets formed from cellular constituents. This is also obvious from the diffusion coefficients measured in our system, as we will discuss below.

3.3. Fluidity of Droplets and Continuous Phase. To characterize the fluidity of the phases, FRAP was used to measure the diffusion coefficient $D$ of the constituent polymer as well as of molecules with varying biological relevance and properties. We used an approach ${ }^{37,38}$ that corrects for diffusion during photobleaching and minimizes error, particularly important when studying fast diffusing molecules (see Figure S4). The measured diffusion coefficient of the small dye Atto647 is overall 5-7-fold higher than that of the labeled Pull$b$-PDMA polymer in water or in any of the phases of the polymer solution, and diffusion slows down by an order of magnitude when the medium is changed from water to polymer solution (Figure 2 and Table S1). The higher diffusivity in the droplets confirms that this is the polymerpoor phase (consistent with the fluorescence signal). The values of the diffusion coefficient do not decrease over time during the measurements (Figure S5) and are very reproducible over a week on droplets from the same batch (here, we display data only for freshly prepared samples), indicating no aging. This is contrary to typical behavior observed, for example, with protein condensate droplets. ${ }^{45-47}$ Compared to the droplets, the recovery of the labeled Pull- $b$ PDMA-Rh is slowed down in the continuous polymer-enriched phase, suggesting higher viscosity. The latter can be roughly estimated from the Stokes-Einstein relation $D=k_{\mathrm{B}} T /(6 \pi \eta a)$ relating the diffusion coefficient of a spherical particle of radius $a$ to the viscosity of the surrounding $\eta ; k_{\mathrm{B}} T$ is thermal energy (note that this approach of assessing the viscosity is very rough considering that the polymer is not a spherical particle). The hydrodynamic radius of the polymer is $4.2 \mathrm{~nm}$ (see Figure S6), yielding a viscosity of the outer polymer-rich phase of $4.12 \pm$ $0.25 \mathrm{mPa} \cdot \mathrm{s}$ and that of the droplet phase of $3.17 \pm 0.33 \mathrm{mPa} \cdot \mathrm{s}$. These values are roughly 4-5 times higher than the viscosity of water and are comparable to viscosities of PEG solutions. ${ }^{48}$ For comparison, protein-rich droplets that phase-separate in cells exhibit viscosities that are $2-3$ orders of magnitude higher
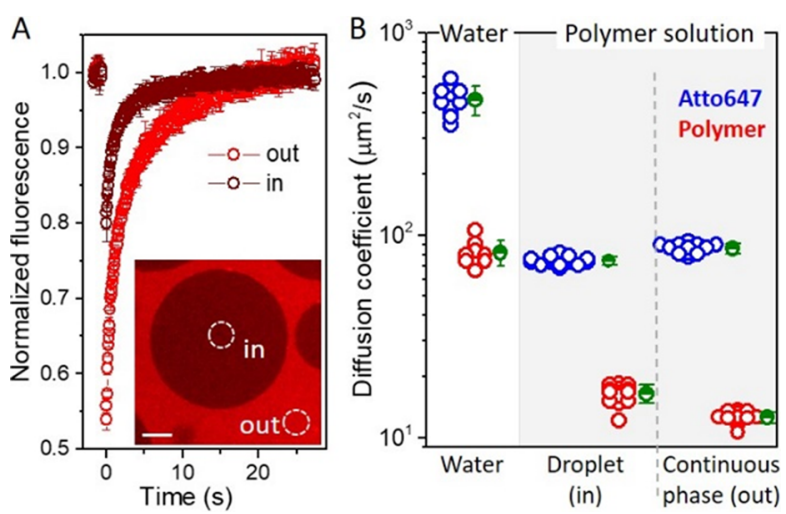

Figure 2. Diffusion coefficient measurements of a small molecule and large molecule in different media. (A) Typical FRAP recovery curves from photobleaching of the labeled Pull-b-PDMA-Rh in the droplet (dark red, in) and the continuous phase (light red, out) of $20 \mathrm{wt} \%$ Pull- $b$-PDMA solution. The average and error are obtained from two consecutive experiments performed on the same spots. The inset shows a typical droplet and the dashed circles indicate the photobleached regions. Scale bar: $10 \mu \mathrm{m}$. (B) Diffusion coefficients measured for Atto647 (blue, $10 \mu \mathrm{M}$ ) and Pull-b-PDMA-Rh (red, 0.02 $w t \%)$ in water and in the polymer solution. Every point corresponds to one measurement. The mean value and standard deviation from different measurements are shown with green half-filled symbols and error bars (see also Table S1).

(740 mPa.s for NPM1 droplets ${ }^{46}$ and $6000 \mathrm{mPa} \cdot \mathrm{s}$ and above for Whi3 droplets ${ }^{47}$ ). Plugging the viscosity of the droplet phase into the above-measured capillary velocity yields for the interfacial tension an extremely low value of the order of 0.05 $\mu \mathrm{N} / \mathrm{m}$. For comparison, interfacial tension measured in PEGdextran aqueous two-phase systems (ATPS) in the low concentration range was reported to approach $0.2 \mu \mathrm{N} / \mathrm{m},{ }^{19}$ which is only slightly higher considering the very rough estimate of the viscosity. Presumably, the diblock nature of Pull-b-PDMA (compared to separate polymers as PEG and dextran) lowers the interfacial tension by suitable polymer arrangement at the interface.

As briefly mentioned above, the test protein Vps24 is enriched in the polymer-depleted droplet phase. Similarly, ATPS systems show preferential partitioning of biological components. Indeed, due to their biocompatibility and the unique feature to provide a mild environment with extremely low interfacial tension, they have been extensively employed for the separation and purification of proteins, nucleic acids, viruses, and cells. ${ }^{18,22}$ Considering the even lower interfacial tensions in our phase-separated polymer solutions and thus potentially higher efficiency, we set out to quantify the partitioning of proteins in our system. The DHBC polymer system explored here possesses a remarkable advantage compared to ATPS: while in ATPSs, the biological component of interest becomes concentrated in a phase rich in one of the constituting polymer species, here, the biomolecules can be enriched in the polymer-depleted phase, potentially allowing an easier purification strategy.

3.4. Partitioning of Small and Large Biomacromolecules: Proteins Are Enriched in the Polymer-Depleted Phase. We aimed at creating a library for the phase preference of a set of fluorescently labeled molecules and particles of varying physical-chemical properties such as size, chemical structure, and charge. The fluorescence signal from the molecules/particles or their analogs was employed to measure 

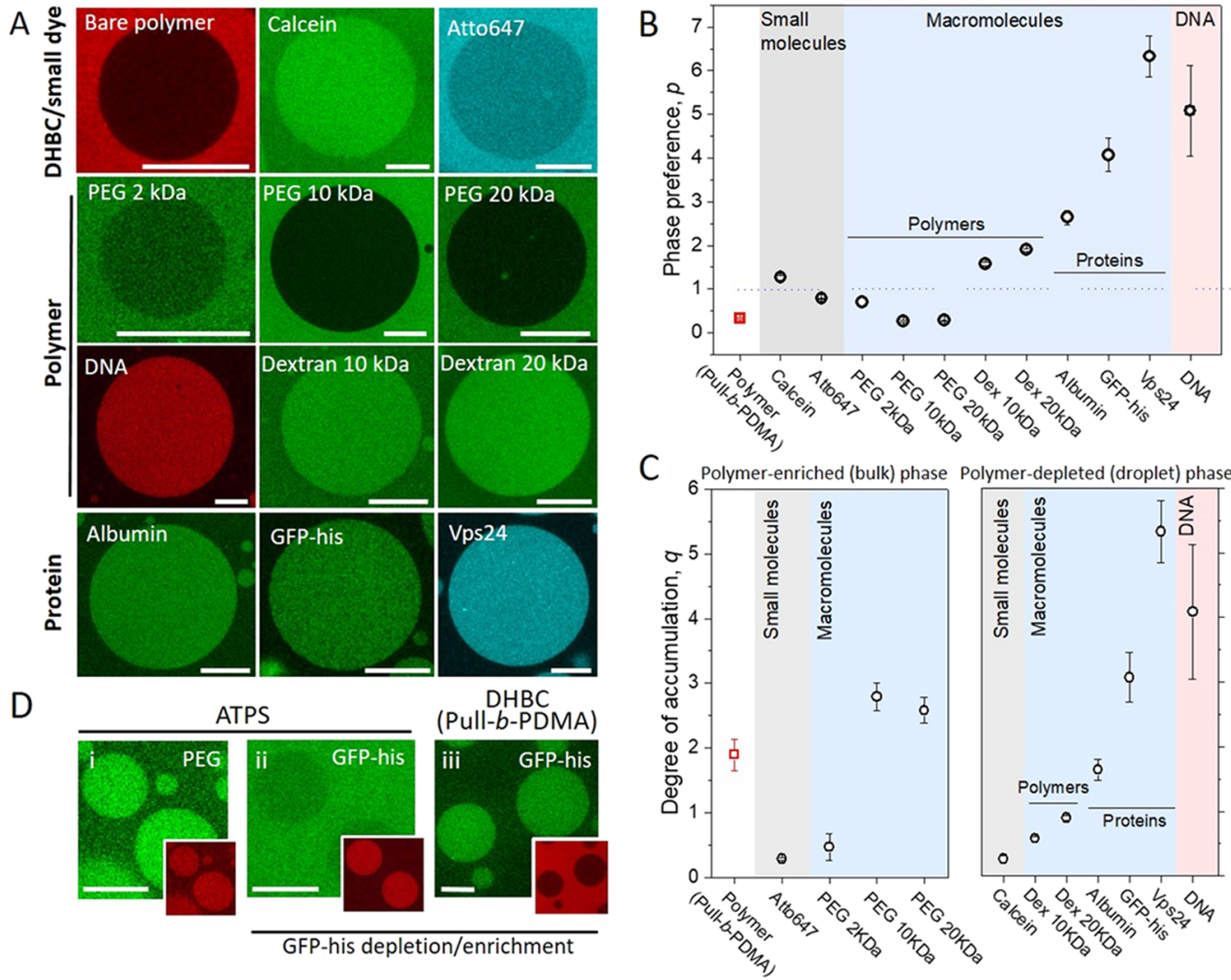

Figure 3. Phase preference for a library of molecules. (A) Confocal images of labeled molecules enriched in one of the two phases. Except for (the red, Cy5-labeled) DNA, all experiments were performed in the presence of $0.02 \mathrm{wt} \%$ labeled Pull-b-PDMA, which was used to confirm that the droplets were the polymer-poor phase (as shown in the first image). Green, red, and blue are pseudocolors for dyes emitting in the green, red, and far-red range. Scale bars: $20 \mu \mathrm{m}$. (B) Average phase preference of the tested molecule measured for a number of droplets $(n>10)$. The concentrations of the molecules investigated for partitioning were as follows: calcein, $10 \mu \mathrm{M}$; Atto647, $10 \mu \mathrm{M} ; 2 \mathrm{kDa}$ PEG, $10 \mu \mathrm{M} ; 10 \mathrm{kDa}$ PEG, 10 $\mu \mathrm{M} ; 20 \mathrm{kDa}$ PEG, $10 \mu \mathrm{M}$; $10 \mathrm{kDa}$ dextran (Dex), $10 \mu \mathrm{M} ; 20 \mathrm{kDa}$ Dex, $10 \mu \mathrm{M}$; albumin, $2 \mu \mathrm{M}$; GFP-his, $10 \mathrm{nM}$; Vps24, $10 \mathrm{nM}$; 6.5-7 kDa DNA, $0.1 \mathrm{nM}$. The macromolecules in the blue-shaded region are arranged in order of increasing molecular weight. The dotted line represents no accumulation. (C) Degree of accumulation (see text for definition) for molecules depleted from (left) or enriched in (right) the droplets. Mean average and standard deviation are shown. (D) Phase preference of GFP-his in the ATPS (4 kDa PEG, 4 wt \%; 400-500 kDa dextran, 5 wt \%) or the DHBC system studied here. The droplets are labeled with $50 \mu \mathrm{M}$ PEG (10 kDa) (i) or $10 \mathrm{nM}$ GFP-his (ii and iii). The labeled Pull-b-PDMA$\mathrm{Rh}(0.02 \mathrm{wt} \%)$ is also used to identify the phases (images in red). Scale bars: $20 \mu \mathrm{m}$.

the partitioning quantitatively. The phase preference $(p)$, or enrichment, is defined as the ratio of fluorescence inside and outside the droplets, $p=I_{\text {in }} / I_{\text {out }}$ (which is equivalent to the socalled partition coefficient defined as the ratio of concentrations in the lighter phase versus the heavier phase ${ }^{18}$ ); $p=1$ corresponds to equal partitioning between the phases, i.e., no preference. The probed materials in the library included (i) small molecules, (ii) macromolecules (polymers and proteins), and (iii) fluid and solid particles. Figure $3 \mathrm{~A}$ comprises of images of droplets in $20 \mathrm{wt} \%$ Pull- $b$-PDMA solution containing the probed materials, and Figure $3 \mathrm{~B}$ shows their phase preference. Some trends are immediately clear: small molecules have a very weak phase preference, with calcein being slightly enriched in the polymer-depleted phase (droplets) and Atto647 being slightly depleted from the droplets. The two tested polymers, PEG and dextran, have opposite preferences; the former is depleted, whereas the latter is enriched in the droplet phase. The opposite preference of PEG and dextran is not surprising considering their wide application as an ATPS. All tested proteins (Vps24, GFP-his, and albumin, with molecular weights ranging from 30 to $\sim 70$ $\mathrm{kDa})$ as well as Cy5-labeled DNA $(6.5-7 \mathrm{kDa})$ have a strong preference for the droplet (polymer-depleted) phase. This demonstrates the potential applicability of our DHBC system for protein and nucleic acid purification. Contrary to reports for size-specific partitioning of DNA in PEG-dextran ATPS systems $^{49}$ and as seen in Figure 3, we did not detect any universal trend for the partitioning of the molecules with respect to molecular weights (the molecular weights are given in Section 2.1) and charge. Presumably, their affinity to the different phases is defined by the affinity to a more or less hydrophilic environment based on the solubility in water as well as interactions with the DHBC itself.

The partition coefficient $p$ does not allow for comparison of the molecule accumulation in the different phases (values lying below 1 show apparent underestimation). To quantify how strong the molecules accumulate (or concentrates) in either phase, we define the degree of accumulation as the ratio of the fluorescence intensity in the phase where it is enriched, $I_{\text {en }}$, over the intensity in the depleted phase, $I_{\text {de }}$, subtracted by 1 : $q=I_{\mathrm{en}} / I_{\mathrm{de}}-1$, which corresponds to the molecular concentration or enrichment gradient. Polymers have very strong enrichment for the given phase, which scales nontrivially with their sizes, and PEG has stronger enrichment compared to dextran of similar sizes (Figure 3C). All three proteins tested have even higher phase enrichment than the 

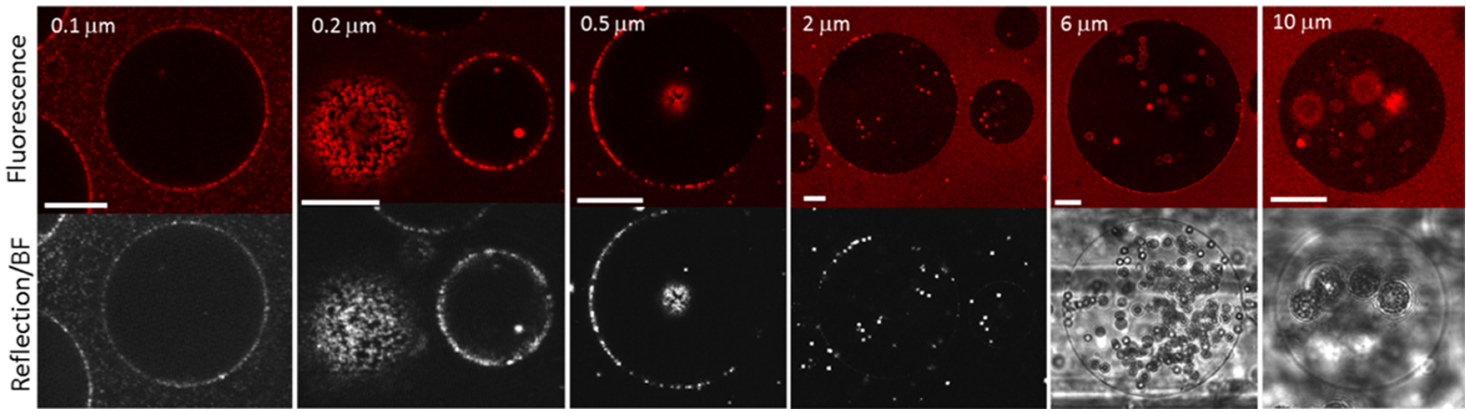

Figure 4. Phase preference of nonlabeled negatively charged poly(styrene) microspheres in phase-separated Pull-b-PDMA (20 wt \%). The upper row shows confocal cross sections ( $0.02 \mathrm{wt} \%$ Pull-b-PDMA-Rh). Lower row shows reflection microscopy (first four snapshots) to image the location of the particles seen as bright spots and bright-field (BF, last two snapshots) images of the same droplets. The numbers on top correspond to particle size. Note that the images for the $0.2 \mu \mathrm{m}$ beads show two droplets, one imaged at its upper surface (left, showing particles adsorbed there) and the other imaged at its equatorial plane (right). Scale bars: $20 \mu \mathrm{m}$.

polymers, with Vps24 being enriched the most, followed by DNA. These results show that physicochemical properties rather than molecular size determine the phase preference. The mechanism of concentrating molecules in a certain phase could be associated with the presumably more hydrophilic environment in the polymer-poor phase (richer in water) compared to the continuous phase, resulting in the accumulation of dextran, DNA, and proteins in the polymer-poor phase.

We next considered the partitioning preference of proteins and the performance of the Pull-b-PDMA as a protein enrichment system compared to a well-established ATPS. As a model protein, we chose GFP-his (which does not show aggregation and is relatively inert with respect to binding to other species) and, as the ATPS, we used PEG ( $8 \mathrm{kDa}, 4 \mathrm{wt} \%)$ and dextran (between 400 and $500 \mathrm{kDa}, 5 \mathrm{wt} \%$ ), the phase separation and binodal of which were studied in more detail in refs 19 and 50. In this ATPS, PEG is enriched in the droplet phase and dextran is enriched in the continuous phase (Figure $3 \mathrm{D}$, i). As a reference, we also used the labeled Pull- $b$-PDMA$\mathrm{Rh}$ polymer, which was found to be enriched in the PEG-rich phase; this could be expected considering that in the DHBC solutions, PEG partitions into the Pull-b-PDMA-rich phase (Figure $3 \mathrm{~A}-\mathrm{C})$. In the ATPS, GFP-his prefers the dextran-rich phase (Figure 3D, ii), which is similar to partitioning observed for other proteins ${ }^{26}$ and could be driven by the phase polarity. ${ }^{51}$ GFP-his preferential partitioning in the ATPS system is rather poor, with a degree of accumulation $q=$ $0.24 \pm 0.06$. In comparison, the degree of accumulation of GFP-his in the Pull-b-PDMA system is around 3 (Figure 3C,D, iii). Although we do not provide an exhaustive comparison of the molecular partitioning in Pull-b-PDMA and PEG-dextran ATPS systems, we already observe that the partition of the model GFP-his protein is more than 10 times better compared to the typical ATPS used for protein purification. In addition, the protein becomes enriched in the polymer-depleted phase (water-rich) rather than in any polymer-rich phase as it is the case for ATPS systems, thus making protein purification less susceptible to polymer contamination, a grand advantage of the Pull-b-PDMA as a potential protein purification system.

3.5. Formation of Protein Condensates. We investigated in more detail the behavior of Vps24, a protein with an extremely high degree of accumulation into the droplet (polymer-depleted) phase (Figure 3C). At $10 \mathrm{nM}$ (bulk) concentration, the droplet contains a few bright fluorescent puncta (see the last image in Figure 3A). As Vps24 does not aggregate in solution at this concentration (aggregation occurs only at high concentrations $>600 \mathrm{nM}),{ }^{52}$ we suspected that the puncta were induced by extensive protein enrichment. In fact, when the bulk concentration is increased to $30 \mathrm{nM}$, the puncta increase in both number and size (Figure S7A), thus confirming that large protein enrichment induces protein crowding and the formation of protein condensates. In other words, the DHBC system can strongly concentrate proteins even at very low overall protein concentration. Interestingly, Vps24 is mobile inside these condensates, as probed by photobleaching them, and the proteins in the condensate are able to exchange with the protein pool from the bulk (Figure S7B), demonstrating that they are fluid similar to protein condensates observed in cells. Thus, extensive protein concentration induced by DHBC phase separation results in strong protein accumulation and crowding, ultimately leading to the formation of a new protein condensate subphase inside droplets that mimics condensate formation even at overall (initial) low protein concentration.

3.6. Particle and Liposome Partitioning: Effect of Surface Charge and Size. Finally, we also studied the phase preference of particles as guest materials of varying size, fluidity, charge, and surface chemistry. As model fluid particles, we used liposomes, whereas as solid particles, we used gold and poly(styrene) beads. We identified cases of preferential partitioning of the particle in the continuous polymer-rich phase, in the droplet polymer-poor phase, and at the domain interface. Neutral and positively charged liposomes (100-200 $\mathrm{nm}$ ) are present exclusively in the continuous phase, whereas negatively charged liposomes are mainly bound to the phase interface (Figure S8A). Similarly, small solid gold nanoparticles $(20-80 \mathrm{~nm})$ are present almost exclusively in the polymer-rich phase (Figure S8B,C).

To study the sole effect of particle size, we used slightly negatively charged poly(styrene) beads of increasing sizes and identical surface chemistry (we assume that the surface charge and chemistry of the particles are the same). We observed a shift in the phase partition with increasing particle size (Figure 4). Small particles $(<0.1 \mu \mathrm{m})$ are mainly located in the continuous phase, intermediate particles $(0.2-2 \mu \mathrm{m})$ are preferably bound to the droplet interface, and larger particles $(>2 \mu \mathrm{m})$ are present exclusively in the droplet phase. This sizephase shift effect is also observed with negatively charged carboxylated poly(styrene) beads (Figure S9; in all cases, Pull$b$-PDMA-Rh appeared to adsorb on the particle surface as observed from the enhanced fluorescence signal on them). However, the preference shift appears to be surface chemistry- 


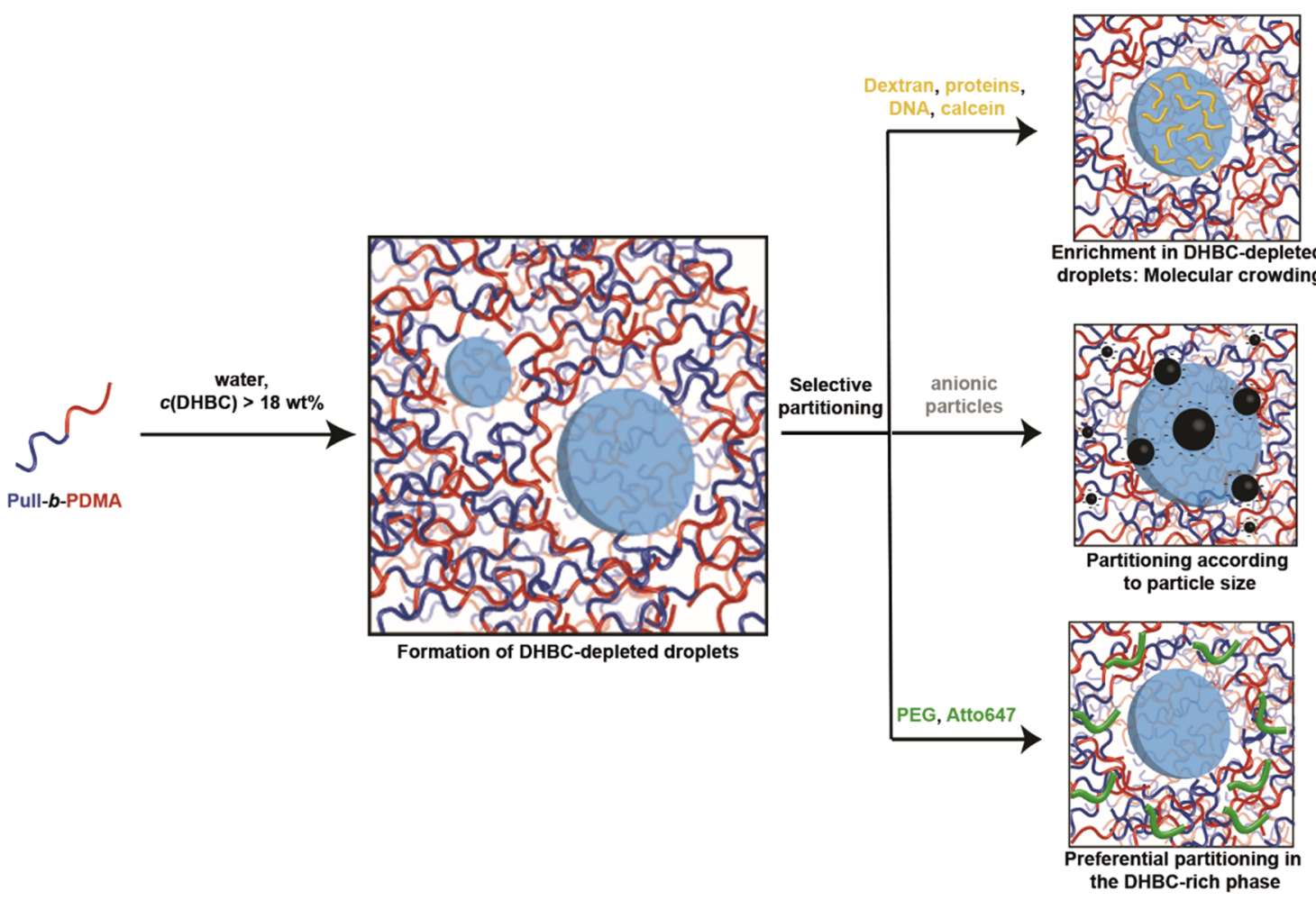

Figure 5. Schematic illustration of the phase separation in solutions of the double-hydrophilic block copolymer Pull- $b$-PDMA and preferential partitioning of various molecules and particles in the water-rich polymer-depleted droplets and in the polymer-enriched continuous phase.

specific and can be reversed depending on particle coating. Small $(2 \mu \mathrm{m})$ streptavidin-coated beads are present in the droplet phase, whereas larger and near-neutral streptavidin and positively charged amino beads $(6 \mu \mathrm{m}$ in size $)$ are both located in the continuous phase (Figure S10), the opposite localization of negatively charged particles of identical sizes. Thus, particle size and surface chemistries, in addition to effects of lowered surface tension, determine particle location in phase-separated Pull- $b$-PDMA polymer phases.

\section{CONCLUSIONS}

Similar to processes of self-coacervation, we observed mesoscale phase separation in DHBC solutions. Intriguing partitioning behavior of macromolecules and particles in phase-separated Pull- $b$-PDMA systems was discovered as schematically summarized in Figure 5. Whereas at low concentration, Pull-b-PDMA is dispersed as a single polymer, at higher concentrations, it phase-separates into two distinct aqueous phases: a more viscous continuous polymer-rich phase and water-rich (polymer-depleted), more fluid phase, and phase separation can be reversed upon dilution and increase in temperature. Depending on the collective properties, such as size, charge, and composition, molecules can be selectively enriched in either phase, with complex molecules exhibiting a much stronger preference compared to simpler molecules. This DHBC system attains a great capacity to preferentially exclude proteins and DNA (and also large particles) from the polymerrich phase.

The DHBC system reported here is reminiscent of the crowded environment in cells in terms of concentration ranges and in terms of phase separation. Phase separation leads to enrichment of macromolecules based on their partition preference and is likely to modulate protein function as exemplified by the observed concentration-dependent aggregation of Vps24 proteins. It will be interesting to study new chemically modified analogs of the DHBC investigated here and examine how these modifications affect the phase in which biopolymers accumulate upon phase separation. Furthermore, it would be interesting to explore higher polymer concentrations, at which the altered interfacial tension might influence protein partitioning.

For the explored DHBC concentration, the polymerdepleted droplets coalesce upon contact and they impose no barrier to the diffusion of water-soluble molecules. The preferred accumulation of various proteins in the Pull- $b$ PDMA-depleted droplets leads to significant molecular crowding. In extreme cases, the enrichment is so efficient that it can increase the local protein concentration above its threshold for condensation in a molecularly crowded environment, leading to the formation of a protein-rich subphase even at initially low protein concentration.

In contrast to most aqueous phase separation systems, which require combining more than one molecular component at specific ratios, ${ }^{43,44,46,47}$ here, the phase separation occurs with a single block copolymer and is driven solely by concentration based on the affinity or solubility of the different blocks in water. The fact that the system presented here contains only one polymer type, compared to at least two (sometimes more) different polymers as in the case in other ATPSs, is advantageous as the number of different polymers as possible contaminants is reduced. In addition, protein enrichment in phase-separated DHBC composed of a single polymer is significantly higher than its competitor, the PEG-dextran ATPS. However, unlike the latter system, proteins in Pull- $b$ PDMA DHBC are located in a polymer-poor phase, reducing contamination. Likewise, these properties can be used to segregate or purify nanometer- and micrometer-sized particles. 
In the future, it would be interesting to investigate the effect of ionic strength and $\mathrm{pH}$ on the stability of the phases and, even more so, the partitioning of various biomacromolecules. For example, it has been shown that several proteins exhibit differences in their phase localization in ATPS due to $\mathrm{pH}$ or salt concentration, ${ }^{18,53,54}$ whereby $\mathrm{pH}$ was even shown to reverse phase localization. Potential improvements could be sought in terms of polymer structure optimization for further improving the polymer partitioning between the phases. ${ }^{51}$ In any case, as demonstrated above, the superiority of DHBC phase separation over ATPS consists of the fact that biomacromolecules become enriched in the polymer-depleted phase; they are enriched better than in an example ATPS, with both of the findings suggesting that DHBCs offer an improved pathway for purification of biomaterials. We also foresee that DHBC could find applications similar to those of ATPS, namely, as crowding reagents for in vitro biochemical investigations, ${ }^{55-57}$ such as those demonstrating crowdinginduced changes in DNA hybridization, ${ }^{58}$ enzyme complex formation, ${ }^{59}$ nanoparticle synthesis, ${ }^{60}$ and protein folding, association, and aggregation. ${ }^{61}$

\section{ASSOCIATED CONTENT}

\section{SI Supporting Information}

The Supporting Information is available free of charge at https://pubs.acs.org/doi/10.1021/acs.macromol.0c01839.

Materials; methods: polymer synthesis, microscopy imaging, FRAP, and dynamic light scattering; table with diffusion coefficients; figures on phase separation, fluidity, and partitioning of molecules and particles (PDF)

\section{AUTHOR INFORMATION}

\section{Corresponding Authors}

Bernhard V. K. J. Schmidt - Max Planck Institute of Colloids and Interfaces, 14424 Potsdam, Germany; School of Chemistry, University of Glasgow, G128QQ Glasgow, U.K.; 10 orcid.org/ 0000-0002-3580-7053; Email: bernhard.schmidt@ glasgow.ac.uk

Rumiana Dimova - Max Planck Institute of Colloids and Interfaces, 14424 Potsdam, Germany; 이이이.org/00000002-3872-8502; Email: rumiana.dimova@mpikg.mpg.de

\section{Authors}

Rafael B. Lira - Max Planck Institute of Colloids and Interfaces, 14424 Potsdam, Germany; Moleculaire Biofysica, Zernike Instituut, Rijksuniversiteit Groningen, 9747 AG Groningen, The Netherlands

Jochen Willersinn - Max Planck Institute of Colloids and Interfaces, 14424 Potsdam, Germany

Complete contact information is available at:

https://pubs.acs.org/10.1021/acs.macromol.0c01839

\section{Notes}

The authors declare no competing financial interest.

\section{ACKNOWLEDGMENTS}

R.B.L. and R.D. acknowledge support from the MaxSynBio consortium, which is jointly funded by the Federal Ministry of Education and Research of Germany and the Max Planck Society. B.V.K.J.S. acknowledges funding from the University of Glasgow. The authors acknowledge Y. Avalos-Padilla, S.
Wegner, J. Steinkühler, and R.L. Knorr for kindly providing us with the Vps24, GFP-his, and labeled DNA samples and J. Zhang for the synthesis of PEG-FITC.

\section{REFERENCES}

(1) Discher, B. M.; Won, Y.-Y.; Ege, D. S.; Lee, J. C.-M.; Bates, F. S.; Discher, D. E.; Hammer, D. A. Polymersomes: Tough vesicles made from diblock copolymers. Science 1999, 284, 1143-1146.

(2) Rideau, E.; Dimova, R.; Schwille, P.; Wurm, F. R.; Landfester, K. Liposomes and polymersomes: a comparative review towards cell mimicking. Chem. Soc. Rev. 2018, 47, 8572-8610.

(3) Antonietti, M.; Förster, S. Vesicles and Liposomes: A SelfAssembly Principle Beyond Lipids. Adv. Mater. 2003, 15, 1323-1333.

(4) Wang, X.; Hu, J.; Liu, G.; Tian, J.; Wang, H.; Gong, M.; Liu, S. Reversibly Switching Bilayer Permeability and Release Modules of Photochromic Polymersomes Stabilized by Cooperative Noncovalent Interactions. J. Am. Chem. Soc. 2015, 137, 15262-15275.

(5) Casse, O.; Shkilnyy, A.; Linders, J.; Mayer, C.; Häussinger, D.; Völkel, A.; Thünemann, A. F.; Dimova, R.; Cölfen, H.; Meier, W.; Schlaad, H.; Taubert, A. Solution Behavior of Double-Hydrophilic Block Copolymers in Dilute Aqueous Solution. Macromolecules 2012, 45, 4772-4777.

(6) Schmidt, B. V. K. J. Double Hydrophilic Block Copolymer SelfAssembly in Aqueous Solution. Macromol. Chem. Phys. 2018, 219, 1700494.

(7) Alarcón, C. d. 1. H.; Pennadam, S.; Alexander, C. Stimuli responsive polymers for biomedical applications. Chem. Soc. Rev. 2005, 34, 276-285.

(8) Gohy, J. F. Stimuli-Responsive Block Copolymer Assemblies. In Block Copolymers in Nanoscience; Lazzari, M.; Liu, G.; Lecommandoux, S., Eds. Wiley: 2006, pp. 91-116, DOI: 10.1002/ 9783527610570.ch5.

(9) Rodríguez-Hernández, J.; Chécot, F.; Gnanou, Y.; Lecommandoux, S. Toward 'smart' nano-objects by self-assembly of block copolymers in solution. Prog. Polym. Sci. 2005, 30, 691-724.

(10) Cölfen, H. Double-Hydrophilic Block Copolymers: Synthesis and Application as Novel Surfactants and Crystal Growth Modifiers. Macromol. Rapid Commun. 2001, 22, 219-252.

(11) Cölfen, H.; Mann, S. Higher-Order Organization by Mesoscale Self-Assembly and Transformation of Hybrid Nanostructures. Angew. Chem., Int. Ed. 2003, 42, 2350-2365.

(12) Taubert, A.; Kübel, C.; Martin, D. C. Polymer-Induced Microstructure Variation in Zinc Oxide Crystals Precipitated from Aqueous Solution. J. Phys. Chem. B 2003, 107, 2660-2666.

(13) Taubert, A.; Palms, D.; Weiss, Ö.; Piccini, M.-T.; Batchelder, D. N. Polymer-Assisted Control of Particle Morphology and Particle Size of Zinc Oxide Precipitated from Aqueous Solution. Chem. Mater. 2002, 14, 2594-2601.

(14) Yu, S.-H.; Antonietti, M.; Cölfen, H.; Giersig, M. Synthesis of Very Thin $1 \mathrm{D}$ and $2 \mathrm{D} \mathrm{CdWO}_{4}$ Nanoparticles with Improved Fluorescence Behavior by Polymer-Controlled Crystallization. Angew. Chem., Int. Ed. 2002, 41, 2356-2360.

(15) Taubert, A.; Furrer, E.; Meier, W. Water-in-water mesophases for templating inorganics. Chem. Commun. 2004, 19, 2170-2171.

(16) Brosnan, S. M.; Schlaad, H.; Antonietti, M. Aqueous SelfAssembly of Purely Hydrophilic Block Copolymers into Giant Vesicles. Angew. Chem., Int. Ed. 2015, 54, 9715-9718.

(17) Hatti-Kaul, R. Aqueous two-phase systems : methods and protocols. Springer Science \& Business Media: 2000.

(18) Albertsson, P. A.. Partition of cell particles and macromolecules: separation and purification of biomolecules, cell organelles, membranes, and cells in aqueous polymer two-phase systems and their use in biochemical analysis and biotechnology; 3rd ed.; John Wiley \& Sons: New York, 1986, p 346 p.

(19) Liu, Y.; Lipowsky, R.; Dimova, R. Concentration Dependence of the Interfacial Tension for Aqueous Two-Phase Polymer Solutions of Dextran and Polyethylene Glycol. Langmuir 2012, 28, 3831-3839. 
(20) Zhang, J.; Hwang, J.; Antonietti, M.; Schmidt, B. V. K. J. Waterin-Water Pickering Emulsion Stabilized by Polydopamine Particles and Cross-Linking. Biomacromolecules 2019, 20, 204-211.

(21) Walter, H.; Brooks, D. E. Phase separation in cytoplasm, due to macromolecular crowding, is the basis for microcompartmentation. FEBS Lett. 1995, 361, 135-139.

(22) Walter, H.; Brooks, D. B.; Fisher, D. Partitioning in aqueous twophase systems: theory, methods, uses, and application to biotechnology; Elsevier: 1987.

(23) Li, Y.; Lipowsky, R.; Dimova, R. Transition from complete to partial wetting within membrane compartments. J. Am. Chem. Soc 2008, 130, 12252-12253.

(24) Dimova, R.; Lipowsky, R. Lipid membranes in contact with aqueous phases of polymer solutions. Soft Matter 2012, 8, 64096415.

(25) Long, M. S.; Cans, A.-S.; Keating, C. D. Budding and asymmetric protein microcompartmentation in giant vesicles containing two aqueous phases. J. Am. Chem. Soc. 2008, 130, 756762.

(26) Long, M. S.; Jones, C. D.; Helfrich, M. R.; Mangeney-Slavin, L. K.; Keating, C. D. Dynamic microcompartmentation in synthetic cells. Proc. Natl. Acad. Sci. U. S. A. 2005, 102, 5920-5925.

(27) Zhao, H.; Ibrahimova, V.; Garanger, E.; Lecommandoux, S. Dynamic Spatial Formation and Distribution of Intrinsically Disordered Protein Droplets in Macromolecularly Crowded Protocells. Angew. Chem. 2020, 59, 11028-11036.

(28) Li, Y.; Kusumaatmaja, H.; Lipowsky, R.; Dimova, R. WettingInduced Budding of Vesicles in Contact with Several Aqueous Phases. J. Phys. Chem. B 2012, 116, 1819-1823.

(29) Li, Y.; Lipowsky, R.; Dimova, R. Membrane nanotubes induced by aqueous phase separation and stabilized by spontaneous curvature. Proc. Natl. Acad. Sci. U. S. A. 2011, 108, 4731-4736.

(30) Liu, Y.; Agudo-Canalejo, J.; Grafmüller, A.; Dimova, R.; Lipowsky, R. Patterns of Flexible Nanotubes Formed by LiquidOrdered and Liquid-Disordered Membranes. ACS Nano 2016, 10, 463-474.

(31) Banani, S. F.; Lee, H. O.; Hyman, A. A.; Rosen, M. K. Biomolecular condensates: organizers of cellular biochemistry. Nat. Rev. Mol. Cell Biol. 2017, 18, 285-298.

(32) Brangwynne, C. P.; Eckmann, C. R.; Courson, D. S.; Rybarska, A.; Hoege, C.; Gharakhani, J.; Jülicher, F.; Hyman, A. A. Germline P Granules Are Liquid Droplets That Localize by Controlled Dissolution/Condensation. Science 2009, 324, 1729-1732.

(33) Avalos-Padilla, Y.; Knorr, R. L.; Javier-Reyna, R.; García-Rivera, G.; Lipowsky, R.; Dimova, R.; Orozco, E. The Conserved ESCRT-III Machinery Participates in the Phagocytosis of Entamoeba histolytica. Front. Cell. Infect. Microbiol. 2018, 8, 53.

(34) Willersinn, J.; Bogomolova, A.; Cabré, M. B.; Schmidt, B. V. K. J. Vesicles of double hydrophilic pullulan and poly(acrylamide) block copolymers: a combination of synthetic- and bio-derived blocks. Polym. Chem. 2017, 8, 1244-1254.

(35) Lira, R. B.; Robinson, T.; Dimova, R.; Riske, K. A. Highly Efficient Protein-free Membrane Fusion: A Giant Vesicle Study. Biophys. J. 2019, 116, 79-91.

(36) Gracià, R. S.; Bezlyepkina, N.; Knorr, R. L.; Lipowsky, R.; Dimova, R. Effect of cholesterol on the rigidity of saturated and unsaturated membranes: fluctuation and electrodeformation analysis of giant vesicles. Soft Matter 2010, 6, 1472-1482.

(37) Kang, M.; Day, C. A.; Kenworthy, A. K.; DiBenedetto, E. Simplified Equation to Extract Diffusion Coefficients from Confocal FRAP Data. Traffic 2012, 13, 1589-1600.

(38) Lira, R. B.; Steinkühler, J.; Knorr, R. L.; Dimova, R.; Riske, K. A. Posing for a picture: vesicle immobilization in agarose gel. Sci. Rep. 2016, 6, 25254.

(39) Johansson, H.-O.; Karlström, G.; Tjerneld, F.; Haynes, C. A. Driving forces for phase separation and partitioning in aqueous twophase systems. J. Chromatogr. B: Biomed. Sci. Appl. 1998, 711, 3-17.
(40) Mika, J. T.; Poolman, B. Macromolecule diffusion and confinement in prokaryotic cells. Curr. Opin. Biotechnol. 2011, 22, 117-126.

(41) Shin, Y.; Brangwynne, C. P. Liquid phase condensation in cell physiology and disease. Science 2017, 357, No. eaaf4382.

(42) Eggers, J.; Lister, J. R.; Stone, H. A. Coalescence of liquid drops. J. Fluid Mech. 1999, 401, 293-310.

(43) Brangwynne, C. P.; Mitchison, T. J.; Hyman, A. A. Active liquid-like behavior of nucleoli determines their size and shape in Xenopus laevis oocytes. Proc. Natl. Acad. Sci. U. S. A. 2011, 108, 4334-4339.

(44) Elbaum-Garfinkle, S.; Kim, Y.; Szczepaniak, K.; Chen, C. C.-H.; Eckmann, C. R.; Myong, S.; Brangwynne, C. P. The disordered P granule protein LAF-1 drives phase separation into droplets with tunable viscosity and dynamics. Proc. Natl. Acad. Sci. U. S. A. 2015, $112,7189$.

(45) Franzmann, T. M.; Jahnel, M.; Pozniakovsky, A.; Mahamid, J.; Holehouse, A. S.; Nüske, E.; Richter, D.; Baumeister, W.; Grill, S. W.; Pappu, R. V.; Hyman, A. A.; Alberti, S. Phase separation of a yeast prion protein promotes cellular fitness. Science 2018, 359, No. eaao5654.

(46) Feric, M.; Vaidya, N.; Harmon, T. S.; Mitrea, D. M.; Zhu, L.; Richardson, T. M.; Kriwacki, R. W.; Pappu, R. V.; Brangwynne, C. P. Coexisting Liquid Phases Underlie Nucleolar Subcompartments. Cell 2016, 165, 1686-1697.

(47) Zhang, H.; Elbaum-Garfinkle, S.; Langdon, E. M.; Taylor, N.; Occhipinti, P.; Bridges, A. A.; Brangwynne, C. P.; Gladfelter, A. S. RNA Controls PolyQ Protein Phase Transitions. Mol. Cell 2015, 60, 220-230.

(48) Gonzalez-Tello, P.; Camacho, F.; Blazquez, G. Density and Viscosity of Concentrated Aqueous Solutions of Polyethylene Glycol. J. Chem. Eng. Data 1994, 39, 611-614.

(49) Nakatani, N.; Sakuta, H.; Hayashi, M.; Tanaka, S.; Takiguchi, K.; Tsumoto, K.; Yoshikawa, K. Specific Spatial Localization of Actin and DNA in a Water/Water Microdroplet: Self-Emergence of a CellLike Structure. ChemBioChem 2018, 19, 1370-1374.

(50) Liu, Y.; Lipowsky, R.; Dimova, R. Giant Vesicles Encapsulating Aqueous Two-Phase Systems: From Phase Diagrams to Membrane Shape Transformations. Front. Chem. 2019, 7, 213.

(51) Iqbal, M.; Tao, Y.; Xie, S.; Zhu, Y.; Chen, D.; Wang, X.; Huang, L.; Peng, D.; Sattar, A.; Shabbir, M. A. B.; Hussain, H. I.; Ahmed, S.; Yuan, Z. Aqueous two-phase system (ATPS): an overview and advances in its applications. Biol. Proced. Online 2016, 18, 18.

(52) Ghazi-Tabatabai, S.; Saksena, S.; Short, J. M.; Pobbati, A. V.; Veprintsev, D. B.; Crowther, R. A.; Emr, S. D.; Egelman, E. H.; Williams, R. L. Structure and Disassembly of Filaments Formed by the ESCRT-III Subunit Vps24. Structure 2008, 16, 1345-1356.

(53) Dominak, L. M.; Gundermann, E. L.; Keating, C. D. Microcompartmentation in Artificial Cells: pH-Induced Conformational Changes Alter Protein Localization. Langmuir 2010, 26, 56975705.

(54) Albertsson, P. Å.; Sasakawa, S.; Walter, H. Cross Partition and Isoelectric Points of Proteins. Nature 1970, 228, 1329-1330.

(55) Ellis, R. J. Macromolecular crowding: obvious but underappreciated. Trends Biochem. Sci. 2001, 26, 597-604.

(56) Zimmerman, S. B.; Minton, A. P. Macromolecular Crowding: Biochemical, Biophysical, and Physiological Consequences. Annu. Rev. Biophys. Biomol. Struct. 1993, 22, 27-65.

(57) Minton, A. P. The Influence of Macromolecular Crowding and Macromolecular Confinement on Biochemical Reactions in Physiological Media. J. Biol. Chem. 2001, 276, 10577-10580.

(58) Goobes, R.; Kahana, N.; Cohen, O.; Minsky, A. Metabolic buffering exerted by macromolecular crowding on DNA-DNA interactions: origin and physiological significance. Biochemistry 2003, 42, 2431-2440.

(59) Rohwer, J. M.; Postma, P. W.; Kholodenko, B. N.; Westerhoff, H. V. Implications of macromolecular crowding for signal transduction and metabolite channeling. Proc. Natl. Acad. Sci. U. S. A. 1998, 95, 10547. 
(60) Plucinski, A.; Willersinn, J.; Lira, R. B.; Dimova, R.; Schmidt, B. V. K. J. Aggregation and Crosslinking of Poly $(\mathrm{N}, \mathrm{N}$-dimethylacrylamide)-b-pullulan Double Hydrophilic Block Copolymers. Macromol. Chem. Phys. 2020, 221, 2000053.

(61) Minton, A. P. Implications of macromolecular crowding for protein assembly. Curr. Opin. Struct. Biol. 2000, 10, 34-39. 